\title{
Extensive cerebral infarction due to involvement both anterior cerebral arteries by Wegener's granulomatosis
}

\author{
J SATOH, ${ }^{1}$ N MIYASAKA,${ }^{2}$ T YAMADA,${ }^{1}$ T NISHIDO,${ }^{1}$ M OKUDA, \\ T KUROIWA, ${ }^{3}$ AND R SHIMOKAWA ${ }^{3}$
}

From the ${ }^{\prime}$ First Department of Internal Medicine, Tokyo Medical and Dental University; the ${ }^{2}$ Division of Clinical Immunology, Institute of Rheumatology, Tokyo Women's Medical College; and the ${ }^{3}$ Department ofo Pathology, Medical Research Institute, Tokyo Medical and Dental University, Tokyo, Japan

SUMmaRY The central nervous system is often affected in Wegener's granulomatosis (WG), butco massive cerebral infarction due to occlusion of branches of the anterior cerebral arteries (ACA) by granulomatous lesions or thrombosis, or both, has seldom been reported. A case is reportedo here of a 67 year old man with WG complicated by generalised necrotising vasculitis in the lung, kidney, and gastrointestinal tract, and cerebral infarction in the territory of both anterior cerebralo arteries, probably caused by thrombosis and a contiguous invasion of granulomatous lesion fromő the nasal cavity.

Key words: bilateral occlusion of anterior cerebral arteries, cerebral vasculitis.

Wegener's granulomatosis (WG) is characterised by systemic necrotising vasculitis and granulomatous lesions. Neurological complications have been estimated to occur in more than half of the patients with WG. ${ }^{1}$ Extensive infarction of both cerebral hemispheres in WG, however, has rarely been recorded. In this report we describe the clinical course and histopathological findings of a case of WG associated with widespread infarction in the region of both anterior cerebral arteries (ACA) attributable to thrombosis due to necrotising vasculitis and direct invasion of the granulomatous lesion from the nasal cavity.

\section{Case report}

A 67 year old man who had been diagnosed as having WG seven years ago was admitted to hospital

Accepted for publication 7 January 1988.

Correspondence to Dr N Miyasaka, Division of Clinical Immunology, Institute of Rheumatology, Tokyo Women's Medical College, Box 116, NS BLD 2-4-1, Nishi-Shinjuku, Shinjuku-ku, Tokyo 163, Japan. because of severe frontal headache on 22 July $1983 . \frac{2}{2}$ One month before admission frontal headacheaccompanied by intermittent fever developed 3 . gradually and persisted. Two days before admission frontal headache became severe and he became? confused and exhibited unusual behaviour. Theo patient had had tuberculous pleuritis at the age of 10 years but since then had been well until seven yearso earlier when nasal obstruction, frequent purulent rhinorrhoea, polyarthralgia, and fever developed or Biopsy of the granulation tissue in the nasal cavity 0 showed a necrotising granulomatous lesion with infiltrates of neutrophils, histiocytes, lymphoid cells, and multinucleated giant cells. While in hospital he suddenly passed a massive bloody stool, and emergency subtotal gastrectomy was performed.? The operative specimen of the stomach showed multiple gastric ulcers associated with necrotisingo vasculitis with fibrinoid necrosis (Fig. 1) and extravascular granulomatous lesions of the submucosa.ब. The wedge biopsy specimen of the liver showed necrotising vasculitis with extravascular granulomas in Glisson's sheath and the subcapsular area.o 


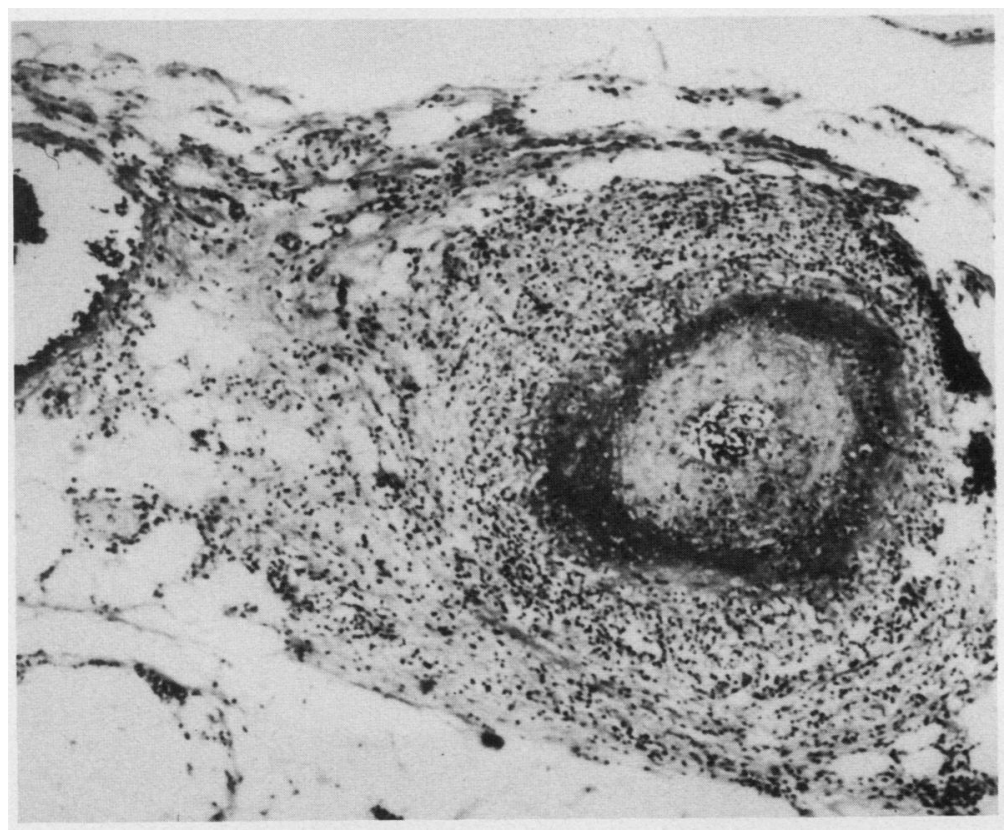

Fig. 1 Operative specimen of the stomach. Necrotising vasculitis with fibrinoid necrosis is prominent. (Haematoxylin and eosin.)

A diagnosis of WG was made, and the patient was transferred to our department. He had proteinuria and microscopic haematuria. Renal biopsy showed focal necrotising glomerulitis with crescents. He was treated with a combination of prednisolone and azathioprine, followed by maintenance treatment with low dose prednisolone and cyclophosphamide. Two short episodes of exacerbation occurred in the following seven years, however. On his own decision the patient took no drugs in the two months before an examination, which showed him to be stuporous and chronically ill. A saddle nose deformity was notable. Nostrils were filled with black crusts. Neurological examination revealed that the left eye had no perception of light; the pupil was dilated and direct light reflex was absent. Other cranial nerves, however, were intact. The neck was supple and the deep tendon reflexes were normal. The urine contained a trace of protein, and was ' ++ ' for glucose; the sediment contained numerous white cells, red cells, and granular casts. Packed cell volume was $0 \cdot 34$; the white cell count was $16 \cdot 8 \times 10^{y} / 1$ with neutrophilia. The blood urea was $10.4 \mathrm{mmol} / \mathrm{l}$, creatinine $114.9 \mu \mathrm{mol} / \mathrm{l}$, glucose $13.5 \mathrm{mmol} / \mathrm{l}$, and serum protein $58 \mathrm{~g} / \mathrm{l}$. The erythrocyte sedimentation rate was $113 \mathrm{~mm} / \mathrm{h}, \mathrm{C}$ reactive protein $4+$, and $\operatorname{IgA}$ $3.23 \mathrm{~g} / \mathrm{l}$. $x$ Ray films of the chest showed a discrete nodular shadow with a thin walled cavity at the left upper zone. The cerebrospinal fluid, with an initial pressure of $350 \mathrm{mmHg}$, contained 34 mononuclear and six polymorphonuclear white cells per $\mathrm{mm}^{3}$; protein was $430 \mathrm{mg} / \mathrm{l}$. Cultures of cerebrospinal fluid were negative. Brain computed tomography (CT) showed a large midline mass $4 \mathrm{~cm}$ in diameter with contrast enhancement in the frontal region adjacent to the falx cerebri (Fig. 2). The lesion was surrounded by extensive perifocal oedema that spread into the frontal white matter on both sides. In addition, the low density area extended to both cingulate gyri. Ventricles were slightly enlarged. Orbital ultrasonography showed a left retrobulbar mass surrounding the optic nerve. Cerebral angiography was not performed because of his poor condition.

The diagnosis considered at that time was intracerebral granulomatous lesion and secondary brain oedema due to WG. The patient was given intravenous infusions of glycerol, $16 \mathrm{mg}$ of dexamethasone, and $100 \mathrm{mg}$ of cyclophosphamide daily, resulting in improvement of the severe headache. Even one month later, however, on neurological examination he was still somnolent, mute, and emotionally labile. The cerebrospinal fluid contained only two mononuclear cells and one polymorphonuclear white cell per $\mathrm{mm}^{3}$ and protein was increased $(3.05 \mathrm{~g} / \mathrm{l})$. An indwelling urinary catheter was inserted because of urinary incontinence and dysuria. About two months after admission the patient developed intermittent fever ranging between 38 and $40^{\circ} \mathrm{C}$. Repeated cultures of urine, 
Fig. 2 Brain CT shows a large midline mass with contrast enhancement adjacent to the falx cerebri. The lesion is surrounded by extensive perifocal oedema spreading into the frontal white matter.

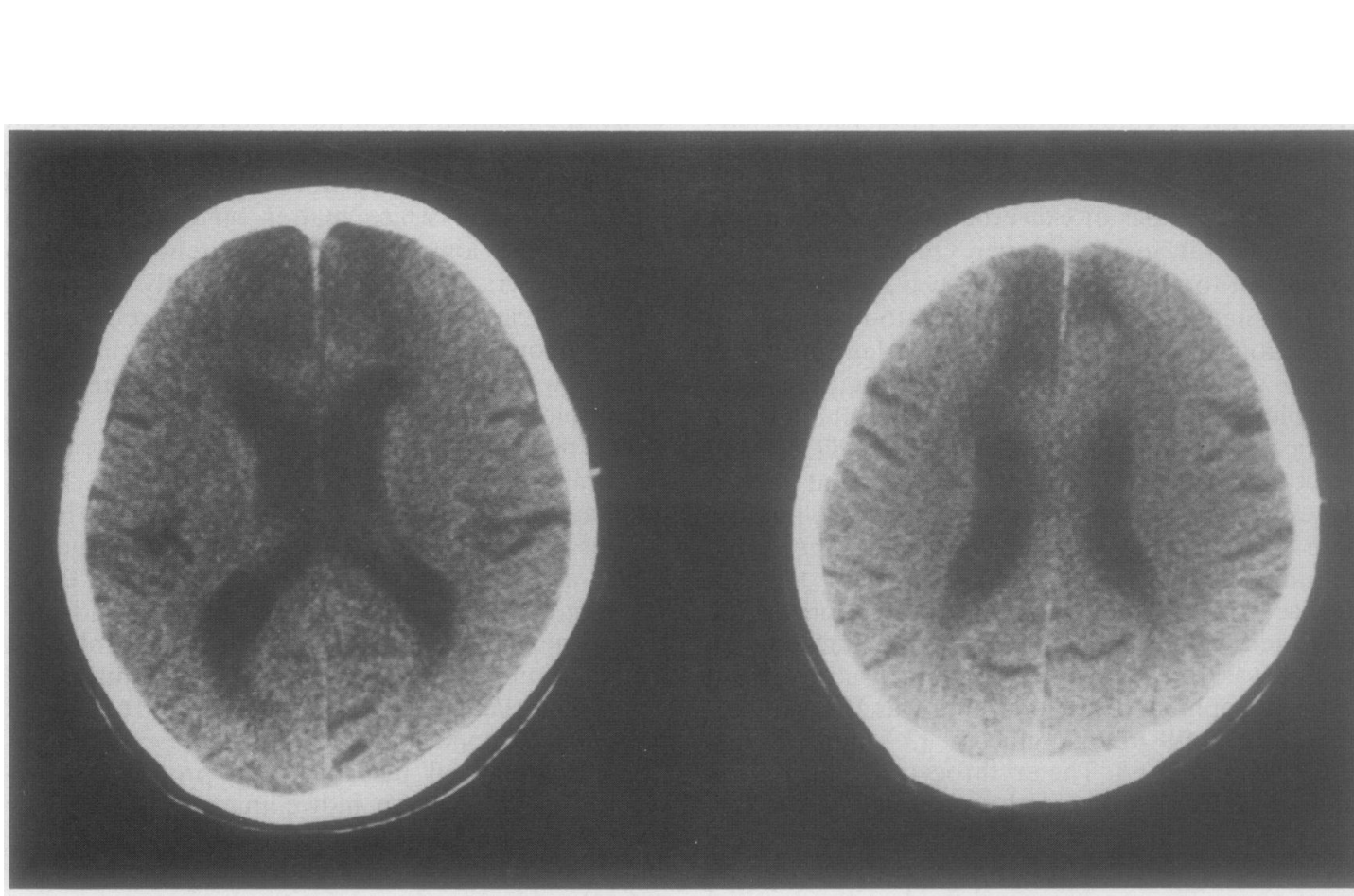

Fig. 3 Brain CT shows significant reduction of the mass lesion adjacent to the falx cerebri. 
sputum, and blood for bacteria and fungi were all negative. Brain CT showed that the midline mass lesion adjacent to the falx cerebri had almost disappeared (Fig. 3). The low density area noticed previously in both cingulate gyri was enlarged, however, indicating the presence of cerebral infarction at the ACA on both sides. The low density area was rimmed with ring enhancement, indicating possible secondary infection in the brain (Fig. 4). He suddenly passed a massive bloody stool, and transfusions of packed red cells and fresh frozen plasma were given. No site of bleeding was identified by colonic endoscopy and mesenteric angiography. He had several attacks of generalised convulsion and died on 13 October 1983.

\section{PATHOLOGICAL. FINDINGS}

General necropsy showed a fist sized, greyish white, soft mass with central cavitation at the left upper lobe, and several scattered foci of greyish white nodules in both lungs. Histologically, these were composed of necrotising vasculitis and granulomas with epithelioid cells, Langhans-type multinucleated giant cells, histiocytes, monocytes, lymphocytes, plasma cells, and neutrophils. Ulcerative lesions were present in the right main bronchus and the posterior wall of the mesopharynx, which were also associated with necrotising vasculitus and granulo- mas. Multiple small greyish white nodules were located at the cortices of both kidneys, and the histology showed focal necrotising glomerulitis, periglomerular granulomatous lesions, and fibrinoid necrosis of the arcuate and interlobular arteries. Multiple shallow ulcers and erosions were scattered in the sigmoid colon and rectum.

Neuropathological examination showed that the brain weighed $1190 \mathrm{~g}$, and the meninges were diffusely clouded and thickened, especially in the frontal poles. Suppurative meningitis of both frontal lobes and the base, involving the left optic nerve, was present. A notable finding was cerebral infarction with symmetrical massive lytic and coagulation necrosis of the area supplied by the branches (A2) of both ACA (Fig. 5). Microscopically, medium to large sized branches of the ACA were occluded with organised mural thrombi, though some of them, were recanalised (Fig. 6). Changes in the vascular walls were not so prominent, but there was some fibrinoid necrosis in these arterial branches of the ACA. By contrast, there were necrotising granulomatous lesions with multinucleated giant cells and various features of necrotising vasculitis of small arteries and veins spread diffusely in the frontal area of the brain base, medial part of the anterior lobes, and meninges, suggesting a contiguous invasion of the granulomatous lesion from the nasal cavity.

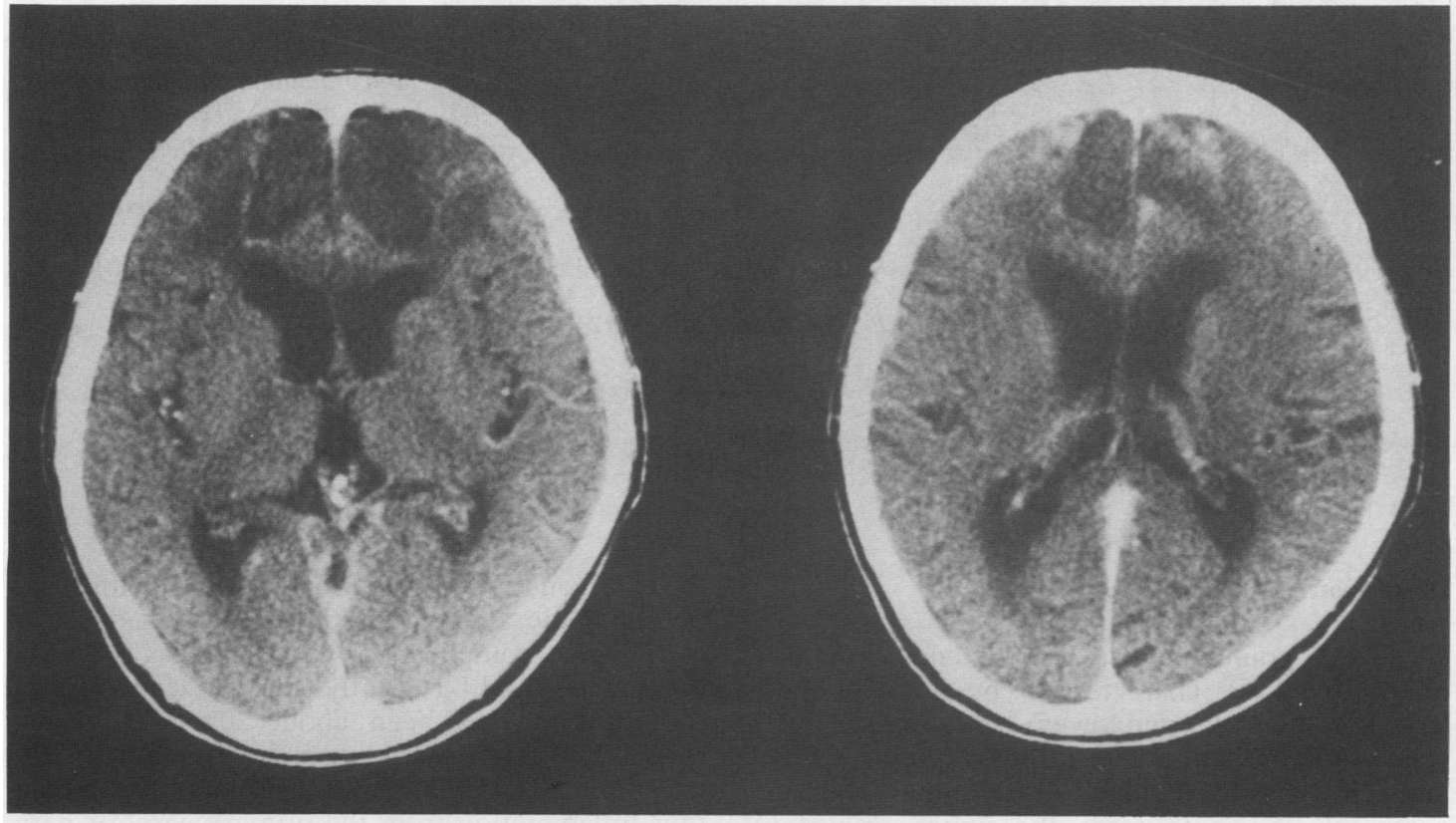

Fig. 4 Brain CT shows enlargement of a low density area with rim enhancement. 


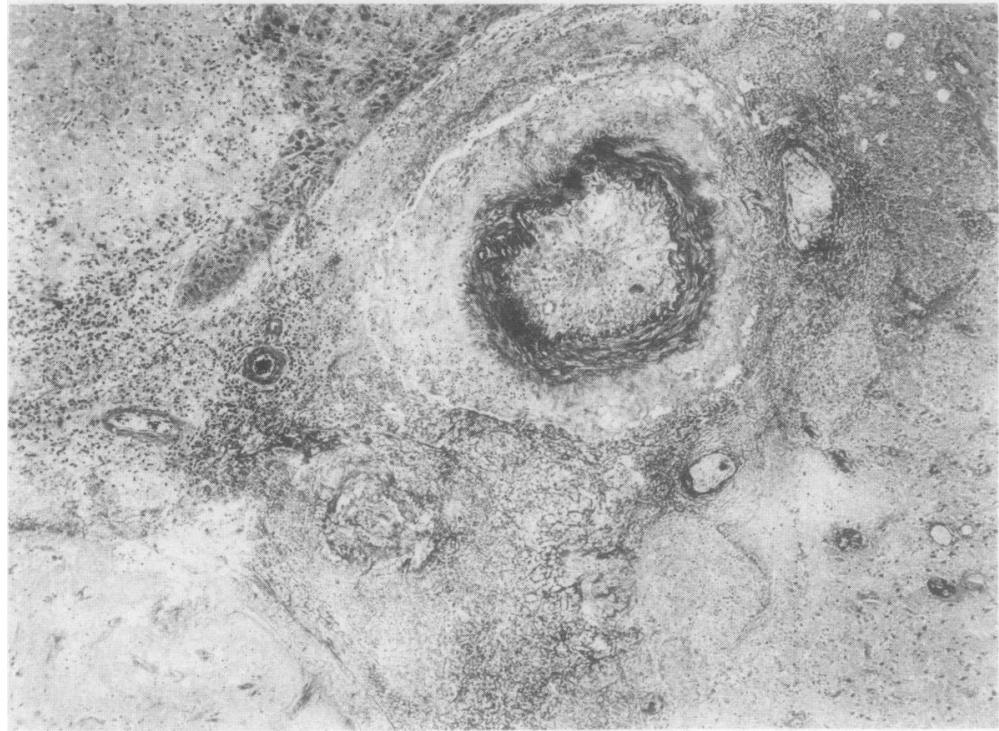

Fig. 5 Necropsy specimen of the medial part of the left anterion lobe shows occlusion of a medium sized branch of the anterior cerebral artery, organise $\vec{d}$ and recanalised. Scattered foci of fresh and old vasculitis of meningeal arteries and veins are $\stackrel{2}{2}$ seen. Foci of lytic and coagulation necrosis of brain parenchyma are also observed. (Haematoxylis and eosin.)

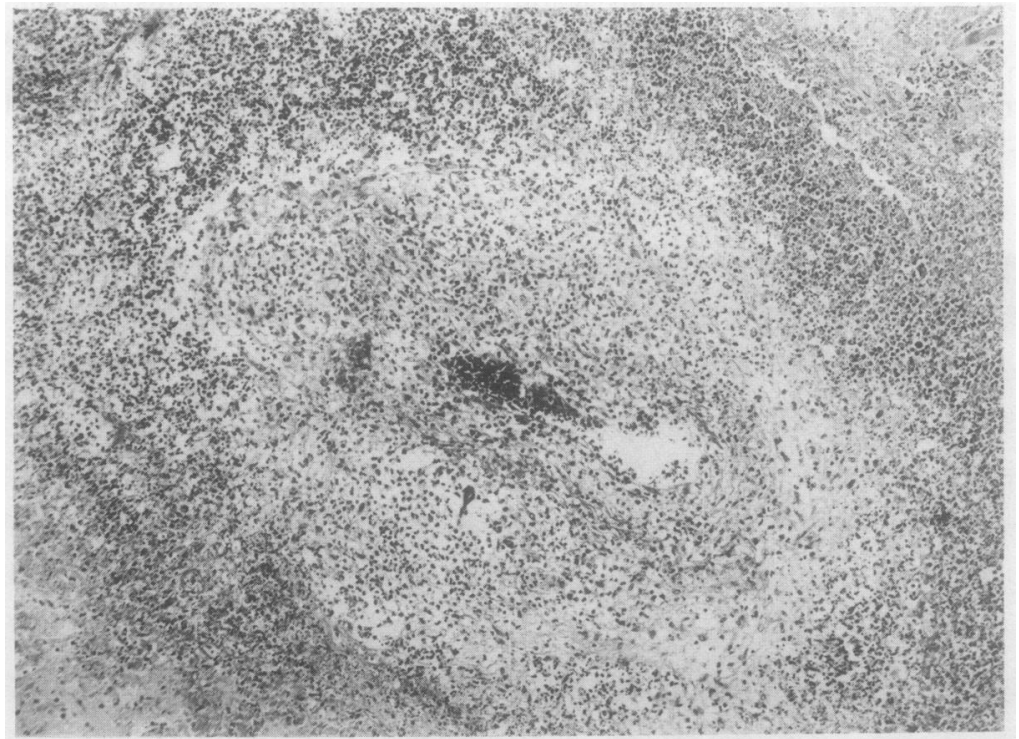

Fig. 6 Necropsy specimen of the medial part of the left anterior lobe shows fresh necrotising and granulomatous panvasculitis of the meningeal artery with infiltration of neutrophils, histiocytes, monocytes, lymphocytes, plasma cells, and multinucleated giant cells. (Haematoxylin and eosin.)

\section{Discussion}

Wegener's granulomatosis is a systemic disease of unknown cause, characterised by granulomatous lesions in the upper and lower respiratory tract with generalised necrotising vasculitis of both arteries and veins, and focal glomerulitis. ${ }^{2}$ Initial symptoms commonly originate from the upper respiratory tract, but multiple organs can be involved. Before the emergence of combination treatment with cyclon phosphamide and corticosteroids the incidence of neurological complications was as high as $50 \%$, ando it could be fatal. ${ }^{1}$ Recently, the incidence of suct

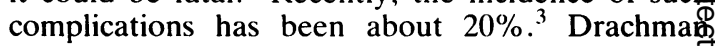
classified the nervous system involvement in W $\vec{\Phi}$ into three forms: contiguous invasion of granulomas tous inflammation from the primary nasal or paranasal lesions, remote granulomatous lesions, an\& 
vasculitis.' Vasculitis of the vasa nervosum is the most common manifestation, and is responsible for cranial and peripheral neuropathies, which take the form of mononeuritis multiplex or polyneuritis. ${ }^{4}$ Vasculitis in the central nervous system is infrequent but may induce cerebrovascular disease ${ }^{15-9}$ namely, subarachnoid or intracerebral haemorrhage, cerebral arterial thrombosis, and sinus thrombosis.

The present case differs from others in the widespread distribution of the infarcted area. Necrotising granulomatous lesions were extensive in the brain base and medial part of the anterior lobes, suggesting a contiguous invasion from the nasal cavity. Furthermore, medium to large sized branches of the ACA were occluded with mural thrombi, which were organised and partly recanalised. We therefore speculate that both thrombosis of the branches of the ACA due to necrotising vasculitis and direct invasion of the granulomatous lesion might have caused cerebral infarction in this case. It is not clear which of these pathological changes is the dominant factor causing cerebral infarction. Clinically, bilateral infarction in the region of the ACA may produce symptoms and signs of frontal lobe dysfunction, e.g., behavioural disturbance, motor inertia, suck and grasp reflexes, incontinence, abulia, muteness, and akinetic mutism. Our case presented neurological deficits that could be attributed to bilateral frontal lobe disturbance.

Immunosuppressive agents, particularly cyclophosphamide, have a dramatic therapeutic efficacy in WG when used in combination with corticosteroids. ${ }^{3}$ Continuous regular monitoring of patients is essential, however, and treatment needs to be continued for a very long time to prevent recurrence of the disease. In the present case the patient had a fatal recurrence of WG after discontinuing treatment, and the administration of cyclophosphamide and dexamethasone could not change the clinical course of the late stage of the disease, though some improvement was evidenced on CT scan. This could be explained by the massive coagulation necrosis of the brain caused by infarction. On the other hand, immunosuppressive treatment can cause opportunistic infection, and suppurative meningitis was observed in this case. The low density area with rim enhancement, as shown in brain CT (Fig. 3), suggested the association of bacterial infection with necrosis of the brain. Cerebrospinal fluid findings also support the association of suppurative meningitis with the late stage of immunosuppressive treatment. It is unlikely that this could contribute to cerebral infarction in the ACA, however.

To our knowledge this is the first clinical and histopathological presentation of extensive infarction of ACA directly caused by occlusion of the vessels, probably resulting from thrombosis due to necrotising vasculitis and direct invasion of extravascular granulomatous lesions from the nasal cavity in WG.

The authors wish to thank Dr Takeshi Tabira of the National Institute of Neuroscience, National Center of Neurology and Psychiatry, and Professor G Palffy of the University of Pecs Medical School. Hungary, for critically reviewing this paper. We also thank Ms Hiromi Kishi for her secretarial work.

\section{References}

1 Drachman D A. Neurological complications of Wegener's granulomatosis. Arch Neurol 1963; 8: 145-55.

2 Godman G C, Churg J. Wegener's granulomatosis. Arch Pathol 1954; 58: 533-53.

3 Fauci A S, Haynes B F, Katz P, Wolff S M. Wegener's granulomatosis: prospective clinical and therapeutic experience with 85 patients for 21 years. Ann Intern Med 1983; 98: 76-85.

4 Moore P M, Cupps T R. Neurological complications of vasculitis. Ann Neurol 1983; 14: 155-67.

5 Fahey J L, Leonard E, Chung J, Godman G. Wegener's granulomatosis. Am J Med 1954; 17: 168-79.

6 Fauci A S, Wolff S M. Wegener's granulomatosis: studies in eighteen patients and a review of the literature. Medicine (Baltimore) 1973; 52: 535-61.

7 Sahn E E, Sahn S A. Wegener's granulomatosis with aphasia. Arch Intern Med 1976; 136: 87-9.

8 Lucas F V, Benjamin S P. Steinberg M C. Cerebral vasculitis in Wegener's granulomatosis. Cleve Clin $Q$ 1976; 43: 275-81.

9 Hearne C B, Zawada E T. Survival after intracerebral hemorrhage in Wegener's granulomatosis. West J Med 1982; 137: 431-4. 